\title{
EEG-Based Drivers' Drowsiness Monitoring Using a Hierarchical Gaussian Mixture Model
}

\author{
Roman Rosipal $^{1}$, Björn Peters ${ }^{2}$, Göran Kecklund ${ }^{3}$, Torbjörn Åkerstedt ${ }^{3}$, \\ Georg Gruber ${ }^{4}$, Michael Woertz ${ }^{1}$, Peter Anderer ${ }^{4,5}$, and Georg Dorffner ${ }^{1,4,6}$ \\ ${ }^{1}$ Austrian Research Institute for Artificial Intelligence, Vienna, Austria \\ ${ }^{2}$ Swedish National Road and Transport Research Institute, Linköping, Sweden \\ ${ }^{3}$ Karolinska Institutet, Stockholm, Sweden \\ ${ }^{4}$ The Siesta Group Schlafanalyse GmbH, Vienna, Austria \\ ${ }^{5}$ Department of Psychiatry and Psychotherapy, Medical University of Vienna, \\ Vienna, Austria \\ ${ }^{6}$ Institute of Medical Cybernetics and Artificial Intelligence, Center for Brain Research, \\ Medical University of Vienna, Vienna, Austria \\ roman.rosipal@ofai.at, bjorn.peters@vti.se, \\ goran.kecklund@ki.se, torbjorn.akerstedt@ipm.ki.se, \\ georg.gruber@thesiestagroup.com, michael.woertz@ofai.at, \\ peter.anderer@meduniwien.ac.at, georg. dorffner@meduniwien.ac.at
}

\begin{abstract}
We developed an EEG-based probabilistic model, which effectively predicts drowsiness levels of thirty-two subjects involved in a moving base driving simulator experiment. A hierarchical Gaussian mixture model (hGMM) with two mixture components at the lower hierarchical level is used. Each mixture models the data density distribution of one of the two drowsiness cornerstones/classes represented by 4-second long EEG segments with low and high drowsiness levels. We transfer spectral contents of each EEG segment into a compact form of autoregressive model coefficients. The Karolinska drowsiness scoring method is used to initially label data belonging to individual classes. We demonstrate good agreement between Karolinska drowsiness scores and the predicted drowsiness, when the hGMM is applied to continuously monitor drowsiness over the time-course of driving sessions. The computations associated with the approach are fast enough to build up a practical real-time drowsiness monitoring system.
\end{abstract}

\section{Introduction}

Drowsiness from combination of sleep loss, high workload prior to driving, and long driving under difficult conditions (night driving, rain, fog, dense traffic) is a significant risk factor that substantially contributes to the number of motor vehicle accidents. For this reason, the drivers' drowsiness has been the subject of intensive research studies followed by the development of the efficient drowsiness monitoring systems. These systems were designed using behavioral (for example, frequency of body movements-actigraph data, steering wheel control), visual (for example, facial 
expression, eyelid movement and closure, head movement, gaze) or physiological (electroencephalogram (EEG), electrooculogram (EOG), electromyogram (EMG)) measures. It has been observed that changes in the human EEG and EOG are very sensitive physiological indicators of drowsiness [11]. The advantage of the EEGbased systems is their high-temporal resolution allowing to track second-to-second drowsiness fluctuations.

However, there is no gold-standard drowsiness scoring system available for scoring EEG/EOG on alert subjects. In this study, the Karolinska drowsiness scoring method [3] was used to visually assign drowsiness levels of 20-second data epochs recorded from subjects participating in a driving simulator experiment.

An EEG-based hierarchical Gaussian mixture model (hGMM) with high-temporal resolution is designed and trained to continuously and automatically monitor/predict drowsiness. The measure of different drowsiness states of subjects participating in a driving experiment are the visually assigned Karolinska drowsiness score (KDS) values. Instead of the strict following of the KDS values, the hGMM is initialized using the information about the extreme drowsiness states-low and high drowsiness cornerstones-only. An output of the model is the continuous curve of the posterior probabilities reflecting a belief about class-membership of the particular data input to the one of the drowsiness cornerstones. Such output representation may become advantageous in the process of building a practical real-time drowsiness monitor when an adaptable threshold triggering a warning system can be used. Finally, a probabilistic framework of the hGMM can be extended in a principled way by considering different sensor modalities and by the inclusion of the contextual/prior information influencing subjects' drowsiness, such as information about the expected physiological state of subjects while driving or about the driving environment itself.

\section{Materials and Methods}

\subsection{Driving Simulator Study}

Procedure: The study was carried out at the Swedish National Road and Transport Research Institute in Linköping (VTI) using the third generation moving base driving simulator, which consists of

- Cut-off passenger car cab

- Computerized vehicle model

- $\quad$ Large moving base system

- Vibration table

- PC-based visual system

- PC-based audio system

The driving simulator is shown in Fig. 1, left. The simulator provides a realistic experimental driving condition that is fully controllable and with a high internal validity (same conditions for all subjects). Furthermore, the simulator makes it possible to carry out safety critical experiments that might be very difficult to do in field studies. The external validity has been found to be very good in previous experiments carried out at VTI [12]. The simulated passenger car had a manual 
gearbox with 5 gears. The time delay introduced in the simulator is very short (max. $40 \mathrm{~ms}$ ), which is important when focusing on the control and maneuvering aspects of driving. The noise, infra-sound and vibration levels inside the cabin corresponded to those of a modern vehicle.

The simulated road had the dimensions and geometry according to a real rural road (Fig. 1, right). The road was $9 \mathrm{~m}$ wide with moderate curves and with a speed limit of $90 \mathrm{~km} / \mathrm{h}$. The driving condition was daylight clear view and full fiction.



Fig. 1. Left: The moving base driving simulator at VTI. Right: A simulated road example.

Subjects: Forty-four shift workers, all non-professional drivers, participated in the experiment. All drove during morning hours directly after a full night-shift with no sleep. Prior to the experiment the subjects received information on how to prepare and they were instructed not to i) drink alcohol for 72 hours before the experiment, ii) drink coffee, tea or other drinks that have an alerting effect for 3 hours before arriving at VTI, iii) eat for 3 hours before arriving at VTI, iv) take a nap during the night shift before the experiment, v) wear make-up when arriving at VTI. All drivers drove at least 45 minutes with a maximum of 90 minutes.

Physiological data: A wide range of data was collected including pre- and postquestionnaires, sleep diary, subjective sleepiness ratings, driving behavior, pupillometry, eye gaze and eye-lid opening, etc. However, in the current work only EEG, EOG, and EMG physiological data were used. Vitaport 2 from Temec Instruments BV was used for all electrophysiological measurements. Vitaport 2 is a portable digital recorder with a hardware-configurable number of channels for physiological measurements. EEG was measured with two monopolar derivations positioned at Fz-A1, Cz-A2 and a bipolar derivation Oz-Pz. Horizontal and vertical EOG movements on both eyes were recorded with three channels. The single EMG electrode was placed under the jaw. The sampling frequency was $256 \mathrm{~Hz}$ for EEG and EMG and $512 \mathrm{~Hz}$ for EOG. Silver cup electrodes were used for all signals. The input data were stored on a flash memory card and were downloaded to a personal computer hard drive for off-line analysis. 


\subsection{Karolinska Drowsiness Scoring Method}

The collected physiological data were used to visually score the drivers' drowsiness level using the Karolinska drowsiness scoring method [3]. The method was developed for quantification of drowsiness in "active" situations (situations when the subject should be awake) and uses EEG and EOG recordings. EMG may be a valuable indicator of arousals and artifacts but is not included among the criteria for drowsiness. The scoring method is based on the Rechtschaffen \& Kales (R\&K) sleep scoring rules [9]. Single channel EEG data from a bipolar Oz-Pz montage were used in the present analysis.

First, the recorded data were divided into 20 -second long epochs. The entire epoch was separated into 2-second bins. The outcome KDS measure described the number of bins in the epoch that contains slow eye movements, alpha activity and/or theta activity; that is, signs of sleepiness. If no sleepiness signs were found, the entire epoch was assigned the KDS value equal to 0 . If one 2-second bin showed signs of sleepiness KDS $=10$ was assigned to the epoch and so on. The maximum KDS level could be 100, which means that the sleepiness signs occur in all 2-second long bins. The epochs with KDS $>50$ were considered to represent "sleep onset". However, according to most definitions of sleepiness, a sleep onset should include theta activity and slow eye movements. The visual scoring was done using the Somnologica® software (Embla). Spectral analysis is an optional step of the scoring method. However, in this study, an advantage of the Fast Fourier transform to examine the temporal patterns and the dynamics of drowsiness on a detailed level was exploited.

\subsection{Hierarchical Gaussian Mixture Model}

The hGMM was used to automatically discriminate and monitor drowsiness. The basic principle of the proposed model architecture is to approximate class-conditional densities of individual drowsiness classes arbitrarily closely. This is achieved with one Gaussian mixture model (GMM) fitted to each class. By defining prior class probabilities, Bayes' theorem can be applied to arrive at posterior probabilities. Thus, the proposed approach can be seen as the extension of Mixture Discriminant Analysis [5] and to be similar to the model called MclustDA [4] (see also [7], [1]). In the current study, the lower hierarchical level of the hGMM consists of two GMMs approximating densities of the low and high drowsiness cornerstones/classes. The upper hierarchical level of the model is then the mixture of these two GMM elements.

The class-conditional distributions at the lower hierarchical level were approximated by GMMs with density function parameters and a number of components being class-specific,

$$
p\left(x \mid C_{i}\right)=\sum_{k \in|C i|} \alpha_{k} p\left(x \mid \theta_{k}\right) \text { with } \sum_{k \in|C i|} \alpha_{k}=1, \quad \alpha_{k} \geq 0
$$

where $x \in R^{d}$ represents a $d$-dimensional observation vector (in this study a vector of the autoregressive coefficients), $\alpha_{k}$ are mixing coefficients, $C_{i,(i=1,2)}$ is the class 
membership index and $\left|C_{i}\right|$ denotes the total number of Gaussian components in each mixture. The Gaussian density function for a given component $k$ has the form

$$
p\left(x \mid \theta_{k}\right)=(2 \pi)^{-d / 2}\left|\Omega_{k}\right|^{-1 / 2} e^{-\left(x-\mu_{k}\right)^{T} \Omega_{k}^{-1}\left(x-\mu_{k}\right) / 2}
$$

where the parameter $\theta_{k}$ consists of a mean vector $\mu_{k}$ and a covariance matrix $\Omega_{k}$.

By applying Bayes' theorem, the posterior probability for a class $C_{i}$ can be calculated as

$$
p\left(C_{i} \mid x\right)=\frac{p\left(x \mid C_{i}\right) p\left(C_{i}\right)}{p(x)} \propto p\left(x \mid C_{i}\right) p\left(C_{i}\right)
$$

where $p\left(C_{i}\right)$ is the non-negative prior class probability. The unconditional density $p(x)$ is not necessarily needed for calculation of the class posteriors, but can be obtained by integrating out the unknown class membership

$$
p(x)=\sum_{i=1,2} p\left(x, C_{i}\right)=\sum_{i=1,2} p\left(x \mid C_{i}\right) p\left(C_{i}\right)
$$

The prior probability $p\left(C_{i}\right)$ has to be non-negative and sum to one considering both classes. In the current study the prior probabilities were not estimated and were set to be equal; that is, $p\left(C_{1}\right)=p\left(C_{2}\right)=1 / 2$.

By fixing the prior probabilities and by training the hGMM on class labeled data, the set of unknown parameters $\Theta_{i}=\left\{\theta_{k}=\left(\mu_{k}, \Omega_{k}\right), \alpha_{k} ; k \in C_{i}\right\}$ can be estimated independently for each Gaussian mixture. This can be done by maximizing the log of the likelihood function

$$
L\left(\Theta_{i} \mid x_{1}, \ldots, x_{N_{i}}\right)=\log \prod_{n=1}^{N_{i}} p\left(x_{n} \mid C_{i}\right)=\sum_{i=1}^{N_{i}} \log \sum_{k \in|C i|} \alpha_{k} p\left(x_{n} \mid \theta_{k}\right)
$$

where $N_{i}$ represents a number of samples from the $i^{\text {th }}$ class used to estimate the parameters of the corresponding Gaussian mixture. A powerful, well-known framework called expectation maximization (EM) was used to maximize the likelihood function with respect to the unknown parameters $\left\{\mu_{k}, \Omega_{k}, \alpha_{k}\right\}$ [2]. Detailed description of the closed form formulas for estimating the individual parameters can by found in [7], [1]. The GMM routines from the Netlab toolbox ${ }^{1}$ (Matlab toolbox for data analysis, [8]) were used to train and test the hGMM.

\subsection{Experiments}

EEG data pre-processing: Prior to building the actual drowsiness models the raw EEG data were pre-processed using the following four steps i) re-sampling the original data to $100 \mathrm{~Hz}$ by using a method that applies an anti-aliasing filter and

\footnotetext{
${ }^{1}$ http://www.ncrg.aston.ac.uk/netlab/
} 
compensates for the filter's delay, ii) band-pass filtering of the re-sampled data into the range of $0.5-40 \mathrm{~Hz}$ by using the $4^{\text {th }}$ order Butterworth filter with zero-phase distortion, iii) windowing of the filtered data into 4-second long non-overlapping segments ${ }^{2}$, iv) computation of the autoregressive (AR) coefficients for each segment. The AR model order was set to 10. If more than one EEG channel was used the AR coefficients were concatenated into a single input vector. These pre-processing steps were found to be useful in previous sleep process modeling studies [10]. A KDS value was assigned to each 4-second segment based on its membership to the corresponding 20 -second segment. Finally, segments containing artifacts visually observed during the Karolinska drowsiness scoring process were removed.

Study A: The performance of the hGMM to classify the EEG epochs with KDS $=0$ and KDS $\geq 50$ was investigated. The purpose of this classification exercise was to demonstrate separability of the two drowsiness cornerstones. Available 4-second data segments of each subject were randomly split into training and testing parts. Ten percent of the segments from each class were kept in the testing part. This procedure was repeated 100 times. The first 20 training partitions were used to determine the number of Gaussian components for each mixture. This was achieved by using the 20 -fold cross-validation method with the validation part representing randomly selected $20 \%$ of each training set. The number of Gaussian components varied in the range 2 to 16 and the final selection was done based on the minimum of the mean classification error computed over 20 validation runs. The hGMM was then trained on all 100 training partitions and the performance was tested on the corresponding testing sets. Using the described validation and testing procedures, all possible combinations of the three EEG electrodes were compared. The aim of this comparison was to determine the influence of different EEG combinations to discriminate between low and high drowsiness.

Study B: In the second step, the hGMM reflecting continuous changes in drowsiness levels was developed. This was achieved using the following strategy. First, 30\% percent of 4-second segments representing the two drowsiness cornerstones were randomly selected from each subject. The hGMM was trained using the selected segments. Next, the posterior values for all data segments arranged in the time-course of the driving experiment were computed. Note that in contrast to the previous classification experiment, also the segments with the KDS values in the range $10-40$ were used and their posterior values were computed. These segments were not used during the training step. For visual purposes the posterior values were re-scaled from their 0 - 1 range into KDS range of 0 - 100. The scaled values were smoothed using a moving average filter. The same smoothing was applied to the KDS values and both smoothed curves were visually compared. The agreement between the smoothed curves was also numerically compared using the nonparametric Spearman rank correlation method [6].

\footnotetext{
${ }^{2}$ This windowing was done in synchrony with 20-second long epochs used for the KDS values assignment. This allows to uniquely determine a KDS value to each 4-second segment based on its membership to the corresponding 20-second epoch.
} 


\section{Results}

Out of the forty-four participants, thirty-two subjects (16 females and 16 males) were considered useful for the current study. Based on visually observed artifacts $18 \%$ of the all 4-second EEG segments were removed resulting in 35295 segments left for the analysis. The histogram of the KDS values of these segments is depicted in Fig. 2. It can be observed that the KDS value equal to 100 was not reached. The number of segments with KDS $=0$ represented $16.8 \%$. Similarly, there were $16.7 \%$ segments with $\mathrm{KDS} \geq 50$ meaning that $66.5 \%$ of the KDS values fell into the range 0 - 50 .

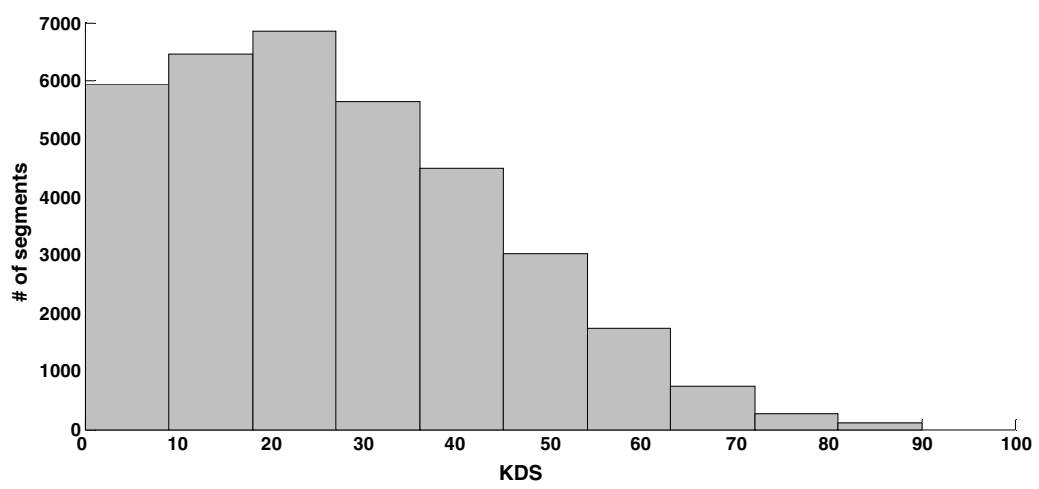

Fig. 2. Histogram of the KDS values for the 4-second segments used in the analysis

Study A: Table 1 summarizes the resulting hGMM test set correct classification rates. The values represent averages over 100 different runs. For each combination of the EEG electrodes, the number of mixture components was found by applying the previously described cross-validation method. Reasonable high correct classification rates can be observed. It is important to note that the KDS values were assigned to 20second long segments while the current hGMM operates on 4-second segments. Furthermore, in contrast to the Karolinska scoring procedure, broadband spectral information from more than one EEG electrode was used in the case of the hGMM.

Table 1. Comparison of the mean and standard deviation of the test set correct classification rates. Values are averaged over 100 runs. The last column represents the number of Gaussian mixture components found by the cross-validation method applied to the first 20 training sets.

\begin{tabular}{|c|c|c|}
\hline EEG electrodes set & $\begin{array}{c}\text { Correct classification rates } \\
\text { Mean ( Standard deviation })\end{array}$ & $\begin{array}{c}\text { Number of Gaussian components } \\
\text { Mixture1 / Mixture2 }\end{array}$ \\
\hline Fz-A1, Cz-A2, Oz-Pz & $77.27(1.65)$ & $4 / 8$ \\
\hline $\mathrm{Cz}-\mathrm{A} 2, \mathrm{Oz}-\mathrm{Pz}$ & $72.90(1.47)$ & $4 / 8$ \\
\hline Fz-A1, Oz-Pz & $76.28(1.21)$ & $6 / 12$ \\
\hline Fz-A1, Cz-A2 & $73.46(1.30)$ & $2 / 6$ \\
\hline $\mathrm{Oz}-\mathrm{Pz}$ & $67.21(1.30)$ & $8 / 16$ \\
\hline $\mathrm{Cz}-\mathrm{A} 2$ & $67.80(1.37)$ & $8 / 10$ \\
\hline $\mathrm{Fz}-\mathrm{A} 1$ & $71.45(1.27)$ & $4 / 12$ \\
\hline
\end{tabular}


The degradation of the performance in comparison to the full-three EEG electrodes-set can be observed when dropping the $\mathrm{Cz}-\mathrm{A} 2$ electrode. The results also suggest the importance of the Fz-A1 electrode inclusion. In Table 2, the confusion tables comparing the performance of the full EEG montage for three different sets of Gaussian mixture components are depicted. In general, very small variability in performance can be observed. Slightly preferable can be the case of using 10 components in each mixture when the most balanced error rates between two classes are observed. Following the results of Tables 1 and 2, the hGMM with 10 Gaussian components in each mixture and using all three EEG electrodes was used in Study B.

Table 2. Confusion tables comparing the performance of the full EEG (Fz-A1,Cz-A2,Oz-Pz) montage for three different sets of mixture components. Results are averages of 100 test sets.

\begin{tabular}{|c|c|c|c|c|c|}
\hline \multicolumn{2}{|c|}{$\begin{array}{c}\text { Mixture1 / Mixture2 } \\
\text { 4 / 8 }\end{array}$} & \multicolumn{2}{c|}{$\begin{array}{c}\text { Mixture1 / Mixture2 } \\
\mathbf{8} \text { / 8 }\end{array}$} & \multicolumn{2}{c|}{$\begin{array}{c}\text { Mixture1 / Mixture2 } \\
\mathbf{1 0} / \mathbf{1 0}\end{array}$} \\
\hline 76.13 & 23.87 & 77.97 & 22.03 & 77.45 & 22.55 \\
\hline 21.57 & 78.43 & 22.97 & 77.03 & 22.69 & 77.31 \\
\hline
\end{tabular}

Study B: After training the hGMM on $30 \%$ of the segments from the two drowsiness cornerstones (KDS $=0$ and KDS $\geq 50$ ), the model was applied to all subjects' data following the time-course of the driving experiment. In general, good visual agreement was observed between the posterior curves representing the predicted drowsiness and the actual KDS values. In Fig. 3 (top plots) the curves of two subjects with the highest Spearman rank correlation coefficient between the smoothed posterior and KDS curves are plotted $(0.81$ - subject 5 and 0.85 - subject 11$)$. The 40-second long moving averaged filter was applied for smoothing the both curves. In three subjects the correlation coefficient values were smaller than 0.2. It is interesting to point out that in these cases the subjects show predominately lower values of KDS with small variability over the whole driving session. An example is plotted in Fig. 3 (bottom left plots, 0.15 - subject 19; note shorter, about 46 min driving in this case). The small posterior values of the prediction model, which are in good agreement with the assigned low drowsiness of the subject (KDS $\leq 30)$, can be seen. The curves of the subject with the value of the correlation coefficient closest to the median correlation equal to 0.53 are plotted in Fig. 3, bottom right plots (0.53 - subject 34). Again, good agreement can be observed between the predicted and actual KDS values.

Finally, the sensitivity of the hGMM to a random selection of the training set and to the EM method convergence was studied. The same training procedure, using $30 \%$ of the two drowsiness cornerstones segments, was repeated 20 times. In the top plot of Fig. 4 an example of 20 curves of the predicted drowsiness of the subject 5 are plotted. Consistent drowsiness prediction can be observed with a low variability among different runs. This is also confirmed with a narrow boxplot representing the distribution of the Spearman rank correlation coefficients computed over 20 runs (bottom plot of Fig. 4). The median value of the correlation coefficients is 0.81 , which 
is in good agreement with a single run case used in Fig. 3. Similarly, the reasonably narrow boxplots for the other three subjects, used in Fig. 3, can be observed in the bottom plot of Fig. 4.
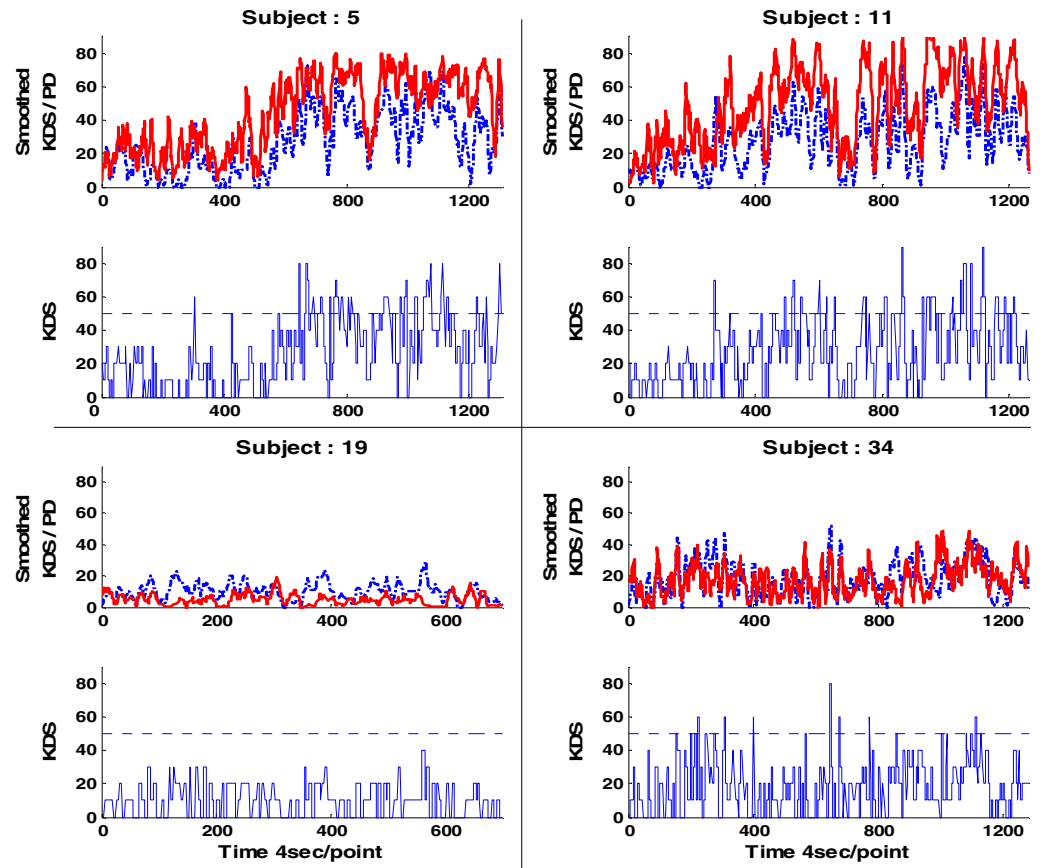

Fig. 3. Top plots: The KDS (dash-dotted) and predicted drowsiness (PD) (solid line) curves. A moving average filter $40 \mathrm{sec}$ long was used. Bottom plots: The non-smoothed KDS values.

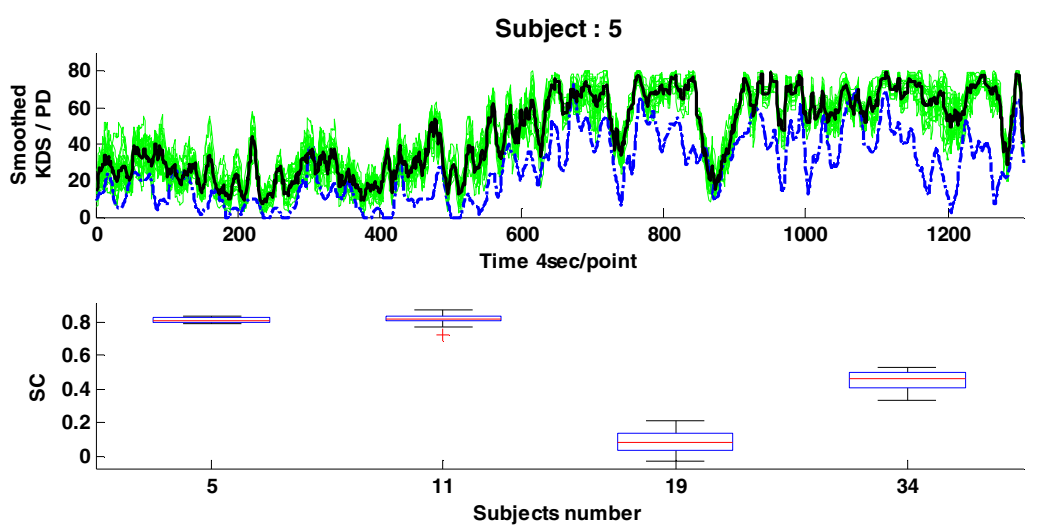

Fig. 4. Top: The KDS (dash-dotted) and twenty predicted drowsiness (PD) (solid lines) curves. The thicker line is the mean value of the PD curves. A moving average filter $40 \mathrm{sec}$ long was used. Bottom: The boxplot of the Spearman rank coefficients (SC) for subjects 5, 11, 19 and 34. 


\section{Conclusions}

The study has shown promising results in applying the presented probabilistic approach to the drivers' drowsiness monitoring task. A reasonably high level of correlation was observed between predicted drowsiness levels and the KDS values. This was despite the fact that the hGMM was applied to shorter data segments, no EOG information was used and, in contrast to the Karolinska scoring protocol, broadband spectral information from multi-electrode EEG setting was considered. The computations associated with the approach are fast enough to build up a practical real-time system. Moreover, such system can be applied and tested in different human drowsiness, vigilance, or fatigue monitoring tasks. Future steps will involve the principled probabilistic inclusion of other physiological signals, visual measures of drowsiness and contextual information into the system. Moreover, implementation of an automatic artifact processing scheme would be a necessary step to build a real-time drowsiness monitoring system.

Acknowledgments. Research supported by the EU project SENSATION/IST 507231. The Austrian Research Institute for Artificial Intelligence is supported by the Austrian Federal Ministry of Education, Science and Culture and the Austrian Federal Ministry of Transport, Innovation and Technology.

\section{References}

1. Bishop, C.: Pattern Recognition and Machine Learning. Springer, Heidelberg (2006)

2. Dempster, A.P., Laird, N.M., Rubin, D.B.: Maximum likelihood from incomplete data via the EM algorithm. J. Royal Stat. Soc. B 39, 1-38 (1977)

3. Gillberg, M., Kecklund, G., Åkerstedt, T.: Sleep and performance of professional drivers in a truck simulator - comparison between day and night driving. J. of Sleep Res. 5, 12-15 (1996)

4. Fraley, C., Raftery, A.E.: Model-Based Clustering, Discriminant Analysis, and Density Estimation. J. Am. Stat. Assoc. 97, 611-631 (2002)

5. Hastie, T., Tibshirani, R.: Discriminant Analysis by Gaussian Mixtures. J. Royal Stat. Soc. B 58, 155-176 (1996)

6. Heckman, N., Zamar, R.: Comparing the shapes of regression functions. Biometrika 87, 135-144 (2000)

7. McLachlan, G.L., Peel, D.: Finite Mixture Models. John Wiley \& Sons, New York (2000)

8. Nabney, I.T.: NETLAB: Algorithms for Pattern Recognition. Springer, Heidelberg (2004)

9. Rechtschaffen, A., Kales, A.: A manual of Standardized Technology, Techniques, and Scoring Systems for Sleep Stages of Human Subjects. Technical Report, Brain Research Institute UCLA, Los Angeles (1968)

10. Rosipal, R., Neubauer, S., Anderer, P., Gruber, G., Parapatics, S., Woertz, M., Dorffner, G.: A continuous probabilistic approach to sleep and daytime sleepiness modeling. ESRS2006, P299, Innsbruck (September 2006)

11. Santamaria, J., Chiappa, K.H.: The EEG of Drowsiness. Demos Medical Publishing (1987)

12. Törnros, J.: Driving behaviour in a real and simulated road tunnel - a validation study. Accident Analysis \& Prevent. 4, 497-503 (1998) 